\title{
Потенциометрическая мультисенсорная система на основе перфторированных мембран и допантов с протоноакцепторными свойствами для определения прокаина и лидокаина в комбинированном препарате
}

\author{
Паршина А.В., Колганова Т.С., Бобрешова О.В. \\ ФГБОУ ВО «Воронежский государственный университет», Воронеж
}

Поступила в редакцию 15.09.2019 г.

DOI: $10.17308 /$ sorpchrom.2019.19/1175

Разработана потенциометрическая мультисенсорная система с перекрестно чувствительными ПД-сенсорами (аналитический сигнал - потенциал Доннана) на основе перфторированных сульфокатионообменных мембран МФ-4СК и Nafion, содержащих 3 мас.\% $\mathrm{SiO}_{2}$, поверхностно модифицированного 10 мол.\% 3-аминопропила, и 2.4 мас.\% $\mathrm{ZrO}_{2}$ для совместного определения катионов прокаина и лидокаина в диапазоне концентраций от $1.0 \cdot 10^{-4}$ до $1.0 \cdot 10^{-2} \mathrm{M}$. Пределы обнаружения ионов прокаина и лидокаина с помощью предложенной мультисенсорной системы составили $1.0 \cdot 10^{-6}$ и $4.5 \cdot 10^{-5} \mathrm{M}$, соответственно. Относительная погрешность и относительное стандартное отклонение определения катионов прокаина в комбинированном фармацевтическом препарате составили 0.5-11 и 4-11\%, катионов лидокаина - 5-6 и 10-12\%, соответственно.

Ключевые слова: мультисенсорные системы, ПД-сенсоры, перфторированные сульфокатионообменные мембраны, гибридные материалы, оксид циркония, оксид кремния, функционализация поверхности допанта, прокаин, лидокаин, фармацевтические препараты.

\section{Potentiometric multisensory system based on perfluorinated membranes and dopants with proton-acceptor properties for procaine and lidocaine determination in combined pharmaceutical}

\author{
Parshina A.V., Kolganova T.S., Bobreshova O.V. \\ Voronezh State University, Voronezh
}

\begin{abstract}
Procaine and lidocaine are widely used in the medical practice both individually and as a part of mixtures for combined local anesthesia. The high performance liquid chromatography and gas chromatography with various detection types are used for the simultaneous determination of local anesthetics in blood plasma, serum, urine, as well as for research of their pharmacokinetics. The chromatographic methods, spectrophotometry, colorimetry, capillary electrophoresis and electrochemical sensors are used for determination of local anesthetics with other active substances or in the presence of their degradation products in pharmaceutical preparations.

The interest in using of hybrid materials in electrochemical sensors for the determination of organic analytes significantly increased in recent years. It was shown previously, that the use of perfluorosulfonic acid cation exchange membranes (MF-4SC and Nafion), modified by zirconia nanoparticles, in the sensors
\end{abstract}


with the Donnan potential (DP) as an analytical signal, allows to reduce the interfering influence of hydroxonium ions present in local anesthetics solutions on their response. This is due to the displacement of electroneutral solution part from the membrane due to the presence of dopant particles in the pores center and due to the electrostatic attraction of the protonated dopant and pore walls. The cross-sensitive DPsensors and their arrays were used for the determination of procaine, lidocaine ions in the individual dosage forms and the model solutions containing potassium chloride.

A potentiometric multisensory system with cross-sensitive DP-sensors based on MF-4SC membrane containing 3 wt.\% of $\mathrm{SiO}_{2}$, surface modified by 10 mol.\% of 3-aminopropyl, and Nafion membrane containing $2.4 \mathrm{wt} . \%$ of $\mathrm{ZrO}_{2}$, is proposed in this work for the simultaneously determination of procaine and lidocaine cations in the concentration range from $1.0 \cdot 10^{-4}$ to $1.0 \cdot 10^{-2} \mathrm{M}$. The limits of detection for procaine and lidocaine ions using the proposed multisensory system were $1.0 \cdot 10^{-6}$ and $4.5 \cdot 10^{-5} \mathrm{M}$, respectively. The relative error of procaine and lidocaine cations determination in a combined pharmaceutical was $0.5-11$ and $5-6 \%$, respectively. The relative standard deviation of procaine and lidocaine cations determination in a combined pharmaceutical was 4-11 and 10-12\%, respectively.

Keywords: multisensory systems, DP-sensor, perfluorosulfonic acid cation exchange membranes, hybrid materials, zirconia, silica, functionalization of dopant surface, procaine, lidocaine, pharmaceuticals.

\section{Введение}

Прокаин и лидокаин широко используются в медицинской практике как индивидуально, так и в составе смесей для проведения комбинированной местной анестезии [1]. В литературе описаны методики для совместного определения местных анестетиков в плазме крови, сыворотке, моче, а также исследования их фармакокинетики, основанные на использовании методов ВЭЖХ [2-6] и ГХ [7] с различными типами детектирования. В фармацевтических препаратах местные анестетики определяют совместно с другими действующими веществами $[8-10,11]$ или в присутствии продуктов их распада [12] с использованием хроматографии, спектрофотомерии, колориметрии, капиллярного электрофореза. Метод мицеллярной жидкостной хроматографии использован для совместного определения лидокаина гидрохлорида и гидрохлорида толперизона в таблетках, растворах для инъекций, кремах и гелях [8]. Авторами [9] разработана спектофотометрическая методика для совместного определения прокаина гидрохлорида и феназона в каплях глазных. В [10] описано экстракционно-спектрофотометрическое определение лидокаина гидрохлорида в инъекциях и спреях. Авторами [11] метод капиллярного электрофореза использован для идентификации и совместного определения прокаина, дигидрострептомицина и пенициллина $\mathrm{G}$ в ветеринарных препаратах. Для определения гидрохлоридов прокаина, бензокаина и лигнокаина в препаратах в присутствии продуктов их разложения, добавок и наполнителей разработана колориметрическая методика, основанная на реакции данных веществ с $n$-бензохиноном в кислой среде с образованием комплексов с переносом заряда [12].

Известны электрохимические сенсоры с различным типом детектирования для определения прокаина и лидокаина в фармацевтических препаратах. В [13] разработан вольтамперометрический графитовый пастовый электрод для определения прокаина в присутствии его метаболита, $n$-аминобензойной кислоты, при контроле качества растворов для инъекций и фармакокинетических исследованиях. Углеродный электрод, полученный по методу трафаретной печати, использован в качестве амперометрического детектора при определении прокаина в препаратах с использованием проточно-инжекционного анализа [14]. Авторами [15] предложены потенциометрические электроды на основе пластифицированных ПВХ-мембран и ионных ассоциатов прокаина и лидокаина с тетрафенилборатом для их определения в твердых и жидких лекарственных формах.

В последние годы значительно вырос интерес к использованию в электрохимических сенсорах для определения органических аналитов гибридных материа- 
лов $[16,17]$. В частности, для определения лидокаина в фармацевтических гелях методами циклической и прямоугольной вольтамперометрии отмечены преимущества допированных бором алмазных электродов по сравнению с немодифицированными углеродными электродами за счет широкого диапазона потенциалов в водных растворах, низкого фонового тока, низкой адсорбции аналита на поверхности и высокой стабильности отклика $[18,19]$. Показано, что использование в сенсоpax, аналитическим сигналом которых является потенциал Доннана (ПД), перфторированных сульфокатионообменных мембран, модифицированных наночастицами диоксида циркония, позволяет снизить мешающее влияние на их отклик ионов гидроксония, присутствующих в растворах местных анестетиков [20]. Это обусловлено вытеснением части электронейтрального раствора из мембраны вследствие присутствия частиц допанта в центре пор и электростатического притяжения протонированного допанта и стенок пор. Перекрестно чувствительные ПД-сенсоры и их массивы использованы для определения ионов прокаина, лидокаина в индивидуальных лекарственных формах и модельных растворах, содержащих хлорид калия [20].

Целью работы явилась разработка потенциометрической мультисенсорной системы с перекрестно чувствительными ПД-сенсорами на основе перфторированных сульфокатионообменных мембран и допантов с протоноакцепторными свойствами для совместного определения ионов прокаина и лидокаина в комбинированном препарате.

\section{Эксперимент}

Объекты исследования. В качестве материалов ПД-сенсоров использовали гибридные материалы на основе перфторированных сульфокатионообменных мембран Nafion и МФ-4СК, предоставленные Лабораторией ионики функциональных материалов ИОНХ РАН (образцы мембран получены к.х.н., старшим научным сотрудником Е.Ю. Сафроновой). Материалы с наночастицами гидратированного $\mathrm{ZrO}_{2}$ были получены на основе экструзионных мембран Nafion и МФ-4СК методом in situ по методике, описанной в [21]. Концентрация $\mathrm{ZrO}_{2}$ в мембранах Nafion coставила 2.0, 2.4, 2.8 мас.\%, а в мембране МФ-4СК - 5.0 мас.\%. Гибридные материалы на основе мембраны МФ-4СК и наночастиц $\mathrm{SiO}_{2}$, поверхностно модифицированных группами 3-аминопропила (R1) и 3-(2-имидазолин-1-ил)пропила (R2), были получены отливкой из раствора полимера в присутствии расчетного количества прекурсора по методике, описанной в [22]. Образцы содержали 3 мас.\% $\mathrm{SiO}_{2}$, концентрация групп R1 и R2 на его поверхности составляли 5 и 10 мол.\% от количества оксида. Допант содержала только $1 / 2$ часть пленки, которая контактировала с исследуемым раствором. Часть пленки, контактирующая с раствором сравнения ПДсенсора, модифицирована не была. Это обеспечивало близость составов раствора внутри мембраны и раствора сравнения ПД-сенсора для нивелирования потенциала Доннана на их границе.

В ПД-сенсорах использовали мембраны в $\mathrm{K}^{+}$-форме. Перевод мембран из протонной в солевую форму снижает влияние на величину и стабильность отклика ПД-сенсора ионов гидроксония, которые имеют высокую подвижность и могут участвовать как в ионообменных, так и в протолитических реакциях в фазах раствора и мембраны. В [23] было показано, что в перфтормембранах полное замещение протонов на катионы местных анестетиков не достигается. Преимущества использования $\mathrm{K}^{+}$-формы мембран обусловлены легким процессом «регенерации» мембран после контакта с полиионными растворами лекарственных веществ. Кро- 
ме того, в этом случае в качестве раствора сравнения для ПД-сенсора используется $1 \mathrm{M}$ раствор $\mathrm{KCl}$. Это обеспечивает наименьшие значения диффузионного потенциала жидкостного соединения на границе раствора сравнения ПД-сенсора с внутренним раствором хлоридсеребряного электрода сравнения.

После длительного использования (до 3 месяцев) мембраны «регенерировали», выдерживая в $2 \mathrm{M}$ растворе $\mathrm{KCl}$ в течение 72 ч, а затем промывали бидистиллированной водой. Между сериями повторных измерений ( 100 измерений) мембраны выдерживали в $0.1 \mathrm{M}$ растворе $\mathrm{KCl}$ в течение 30 мин при постоянном перемешивании и хранили в бидистиллированной воде. Между измерениями мембраны помещали в бидистиллированной воду.

Для приготовления исследуемых растворов использовали сухие вещества прокаина гидрохлорида (РroHCI, 2-(диэтиламино)этил-4-аминобензоат, SigmaAldrich, 99\%), лидокаина гидрохлорида (LidHCI, 2-(диэтиламино)-N-(2',6'диметилфенил)ацетамид, Sigma-Aldrich, 99\%), растворы для инъекций «Новокаин» (ОАО «Дальхимфарм») и «Лидокаин» (ОАО «Мосхимфарм-препараты» им. Н.А. Семашко»), бидистиллированную воду (удельное сопротивление 18 МОм.см).

Водные растворы, содержащие ProHCI и LidHCI с различным соотношением концентраций в диапазоне от $1.0 \cdot 10^{-4}$ до $1.0 \cdot 10^{-2} \mathrm{M}(\mathrm{pH} 4.06-6.09)$, готовили растворением точных навесок сухих веществ в бидистиллированной воде. Растворы препарата готовили из растворов для инъекций в различном объемном соотношении с последующим разбавлением бидистиллированной водой. Концентрация действующих веществ в препаратах «Новокаин» и «Лидокаин» составляет 20 и $100 \mathrm{Mг} / \mathrm{cm}^{3}$, что соответствует концентрации ионов $\operatorname{ProH}^{+}$и $\mathrm{LidH}^{+} 0.0733$ и $0.3693 \mathrm{M}$, соответственно. Вспомогательными веществами «Новокаина» являются $0.1 \mathrm{M}$ раствор $\mathrm{HCl}$ (до $\mathrm{pH} 3.8-4.5$ ) и вода для инъекций, а «Лидокаина» - 1 М раствор $\mathrm{NaOH}$ и вода для инъекций.

Методы исследования. Ячейка для оценки откликов системы ПД-сенсоров включала два корпуса из непроводящего материала, набор мембран разного состава, хлоридсеребряные электроды и многоканальный потенциометр. Внутренний корпус $(\mathrm{d}=4.5 \mathrm{~cm}, \mathrm{~h}=3.5 \mathrm{~cm})$ заполнялся исследуемым раствором. Внешний корпус включал одну секцию $(\mathrm{d}=5 \mathrm{~cm}, \mathrm{~h}=3 \mathrm{~cm})$ для внутреннего корпуса и восемь секций $\left(\mathrm{V}=28 \mathrm{~cm}^{3}\right)$ для раствора сравнения $(1 \mathrm{M} \mathrm{KCl})$. Мембраны одним концом погружали в исследуемый раствор, а другим концом - в одну из секций с раствором сравнения. При использовании гибридных мембран в исследуемый раствор погружали конец модифицированной части образца. Хлоридсеребряный электрод (ЭСр-10103, ООО «Эконикс-эксперт»), подключенный к входу многоканального потенциометра для электрода сравнения, погружали в исследуемый раствор, а хлоридсеребряные электроды (ЭСр-10103, ООО «Сенсорные Системы»), подключенные к измерительным входам, - в секции с раствором сравнения. Напряжение нескольких цепей $(\mathrm{Ag}|\mathrm{AgCl}, 1 \mathrm{M} \mathrm{KCl}|$ мембрана $\mid$ исследуемый раствор $\mid$ нас. $\mathrm{KCl}, \mathrm{AgCl} \mid \mathrm{Ag})$ измеряли по очереди с помощью многоканального аналого-цифрового преобразователя. Одновременно с этим измеряли $\mathrm{pH}$ исследуемого раствора с применением стеклянного электрода (ЭС-10301/4, ООО «Эконикс-эксперт»).

Расчёт коэффициентов градуировочных уравнений методом многомерного регрессионного анализа выполняли с учетом влияния на отклик ПД-сенсоров концентрации ионов $\mathrm{ProH}^{+}, \mathrm{LidH}^{+}$и $\mathrm{H}_{3} \mathrm{O}^{+}$:

$$
\Delta \varphi_{\mathrm{D}}=\mathrm{b}_{0}+\mathrm{b}_{1} \mathrm{pProH}+\mathrm{b}_{2} \mathrm{pLidH}+\mathrm{b}_{3} \mathrm{pH}
$$

где $\Delta \varphi_{\mathrm{D}}$ - величина отклика ПД-сенсора, $\mathrm{MB}$; pProH - отрицательный десятичный логарифм молярной концентрации ионов $\mathrm{ProH}^{+}$; $\mathrm{pLidH}$ - отрицательный десятич- 
ный логарифм молярной концентрации ионов $\mathrm{LidH}^{+} ; \mathrm{b}_{0}-$ свободный член градуировочного уравнения, мB; $\mathrm{b}_{\mathrm{i}}-$ коэффициенты чувствительности ПД-сенсора к соответствующим ионам, мB/pc. Для выявления возможных систематических ошибок и доказательства правильности выбора градуировочного уравнения оценивали его адекватность по F-критерию Фишера. Значимость коэффициентов уравнения оценивали по t-критерию Стьюдента.

Для оценки воспроизводимости отклика ПД-сенсора в исследуемом растворе определяли дисперсию отклика $\left(\mathrm{s}^{2}, \mathrm{mB}^{2}\right)$. Оценку стабильности откликов ПДсенсоров выполняли на основе результатов хронопотенциометрических измерений в течение 1 ч. Для определения времени отклика сенсора $\left(\mathrm{t}_{\mathrm{resp}}\right.$, мин) сравнивали разброс значений отклика в течение времени измерения с разбросом значений при дублировании эксперимента. Дрейф отклика сенсора определяли как его изменение в единицу времени (мВ/ч) после установления отклика.

Пределы обнаружения $\left(\mathrm{c}_{\min }\right)$ аналитов оценивали по правилу «три сигма» как их минимальную концентрацию, при которой величина отклика сенсора в растворе $\left(\Delta \varphi_{\min }\right)$ отличается от величины отклика в фоне $\left(\Delta \varphi_{\text {фон }}\right)$ более, чем на утроенное стандартное отклонение отклика в фоне $\left(\mathrm{s}_{\text {фон }}, \mathrm{MB}\right)$. В качестве фона использовали бидистиллированную воду.

Корреляцию между откликами пар ПД-сенсоров для оценки возможности объединения их в массив оценивали по r-критерию.

Для расчета концентраций аналитов в растворе и фармацевтическом препарате решали систему двух градуировочных уравнений для выбранной пары ПДсенсоров. Экспериментальными данными для расчета концентраций были значения откликов ПД-сенсоров и $\mathrm{pH}$ в объекте анализа. Относительную погрешность $\left(\delta=\left(\mathrm{c}_{\text {эксп }}-\mathrm{c}_{\text {теор }}\right) / \mathrm{c}_{\text {теор }}, \%\right)$ и относительное стандартное отклонение $\left(\mathrm{s}_{\mathrm{r}}=\mathrm{s} / \mathrm{c}_{\text {эксп }}, \%\right)$ pacсчитывали для оценки правильности и воспроизводимости определения аналитов.

\section{Обсуждение результатов}

ПД-сенсоры на основе мембран $\mathrm{Nafion}_{\text {(экструзия, in situ) }}+\mathrm{ZrO}_{2}$,

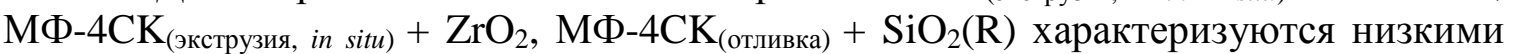
значениями времени установления ( $<1$ мин), дрейфа $(\leq 6 \mathrm{MB} / 4)$ и дисперсии (10$21 \mathrm{MB}^{2}$ ) отклика в растворах ProHCI+LidHCI в диапазоне концентраций $1.0 \cdot 10^{-4}$ $1.0 \cdot 10^{-2} \mathrm{M}$ при $\mathrm{pH}$ 4.06-6.09. Градуировочные характеристики ПД-сенсоров не изменялись в течение 1 года использования. Это обусловлено тем, что при переводе мембран в $\mathrm{K}^{+}$-форму регенерация происходит полностью.

Чувствительность ПД-сенсоров к ионам $\mathrm{ProH}^{+}$и $\mathrm{LidH}^{+}$возрастает для ряда мембран Nafion ${ }_{\text {(экструзия) }}<\mathrm{M} \Phi-4 \mathrm{CK}_{\text {(экструзия) }}<\mathrm{M} \Phi-4 \mathrm{CK}_{\text {(отливка) }}$ (2)-(4). При этом чувствительность ПД-сенсоров к ионам $\mathrm{LidH}^{+}$возрастает сильнее, достигая более высоких значений, чем к ионам $\mathrm{ProH}^{+}$при использовании мембраны МФ-4СК (отливка) $(4)$. Тогда как при использовании экструзионных мембран чувствительность к ионам $\mathrm{LidH}^{+}$ниже, чем к ионам $\mathrm{ProH}^{+}(2)$, (3). Полученные результаты согласуются с результатами исследования сорбции ионов $\mathrm{ProH}^{+}$и $\mathrm{LidH}^{+}$из растворов ProHCI и LidHCI мембранами, полученными отливкой и экструзией [23]. Видимо, сорбция ионов $\mathrm{LidH}^{+}$, имеющих гидрофобный радикал, мембранами Nafion (экструзия) приводит к гидрофобизации мембраны и дополнительному уменьшению объема внутри пор. Увеличение же размера пор и неоднородности мембран МФ-4СК, особенно полученных отливкой, облегчает переход в нее объемных катионов и их концентрация в порах определяется размером, который у ионов $\mathrm{LidH}^{+}$меньше. При этом для всех немодифицированных образцов наблюдалась высокая чувствительность 
ПД-сенсоров к мешающим ионам $\mathrm{H}_{3} \mathrm{O}^{+}$(2)-(4). Поэтому модификация мембран была направлена на ее снижение.

$$
\begin{aligned}
& \Delta \varphi_{\mathrm{D}}=120 \text { - 15.8·pProH - 8.9.pLidH - 42.2·pH, Nafion }{ }_{\text {(экструзия) }} \\
& \Delta \varphi_{\mathrm{D}}=43 \text { - 17.9.pProH - 9.0.pLidH - 16.6·pH, МФ-4СК (экструзия) } \\
& \Delta \varphi_{\mathrm{D}}=58 \text { - 26.7·pProH - 47.4.pLidH - 32.5·pH, MФ-4CК (отливка) }
\end{aligned}
$$

Способ получения гибридных мембран также существенно влияет на чувствительность ПД-сенсоров к аналитам: ее значения к ионам $\mathrm{ProH}^{+}$и $\mathrm{LidH}^{+}$для мембран Nafion (экструзия, in situ) и МФ-4СК (экструзия, in situ), с частицами $\mathrm{ZrO}_{2}$ являются менее высокими (рис. 1), чем для мембран МФ-4СК (отливка), содержащих частицы $\mathrm{SiO}_{2}(\mathrm{R})$ (рис. 2). Для всех гибридных мембран на основе экструзионных образцов чувствительность ПД-сенсоров к ионам $\mathrm{LidH}^{+}$ниже, чем к ионам $\mathrm{ProH}^{+}$, а для мембран, полученных отливкой, соотношение обратное.

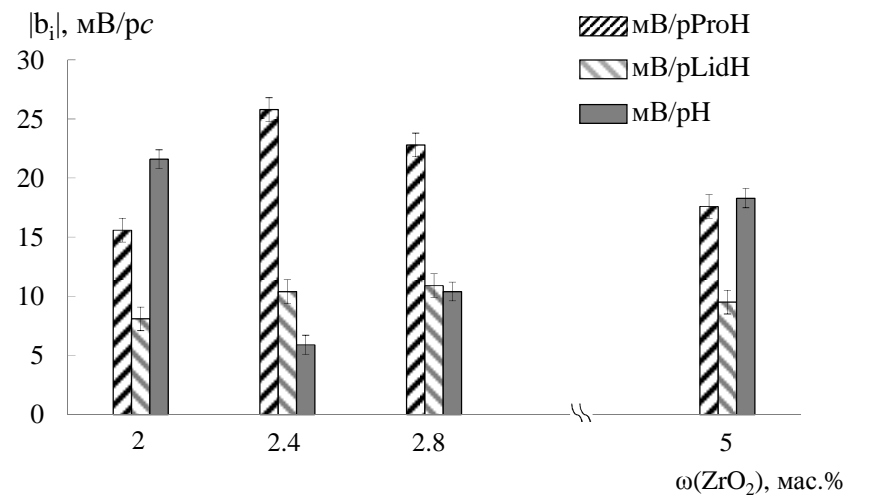

Рис. 1. Коэффициенты чувствительности ПД-сенсоров на основе мембран Nafion $_{\text {(экструзия, in situ) }}$ и МФ-4СК (экструзия, in situ), содержащих $\mathrm{ZrO}_{2}$, к катионам в растворах ProHCI+LidHCI

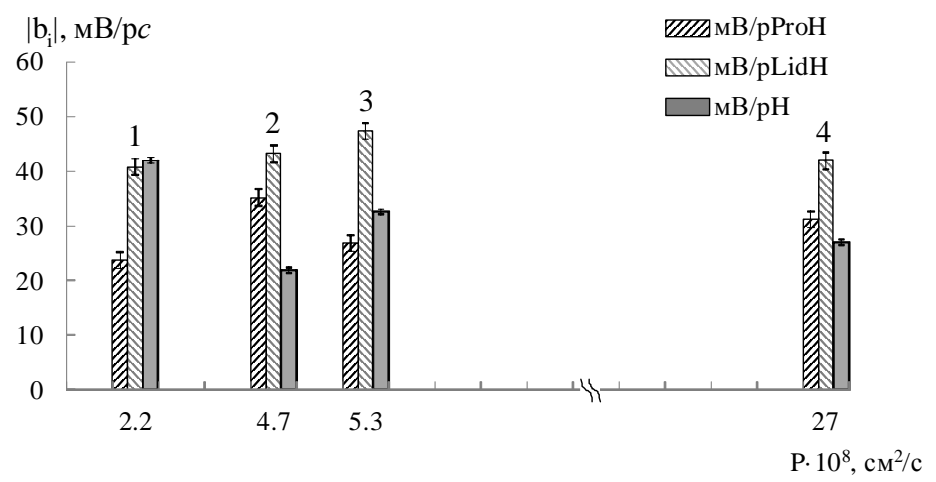

Рис. 2. Зависимость коэффициентов чувствительности ПД-сенсоров к катионам в растворах ProHCI+LidHCI от диффузионной проницаемости мембран $\mathrm{MФ-4CK}$ (олливка) +3 мас.\% $\mathrm{SiO}_{2}(\mathrm{R}): 1$ - 5 мол.\% $\mathrm{R} 2 ; 2$ - 5 мол.\% R1;

$$
3 \text { - исходный образец; } 4 \text { - } 10 \text { мол.\%R1 }
$$

Для гибридных мембран обоих типов, наблюдалось влияние размера частиц, вводимых в поры, на чувствительность ПД-сенсоров к ионам $\mathrm{ProH}^{+}, \mathrm{LidH}^{+}$и $\mathrm{H}_{3} \mathrm{O}^{+}$ (рис. 1,2$)$. Для мембран МФ-4CK (отливка) $+\mathrm{SiO}_{2}(\mathrm{R})$ в качестве косвенной характеристики размеров внутрипорового пространства использовали значения диффузионной проницаемости мембран, изученной для данных образцов в работе [22]. При использовании экструзионных мембран наибольшая чувствительность ПДсенсоров к ионам $\mathrm{ProH}^{+}, \mathrm{LidH}^{+}$и при этом наименьшая к ионам $\mathrm{H}_{3} \mathrm{O}^{+}$достигалась при концентрации 2.4-2.8 мас.\% $\mathrm{ZrO}_{2}$ (рис. 1). Снижение чувствительности ПДсенсоров к ионам $\mathrm{H}_{3} \mathrm{O}^{+}$также наблюдалось при использовании образца МФ- 
$4 \mathrm{CK}_{\text {(отливка) }}+3$ мас.\% $\mathrm{SiO}_{2}(5$ мол.\% $\mathrm{R} 1)$, характеризующегося пониженной диффузионной проницаемостью (рис. 2). Видимо, при этих условиях переход объемных катионов в мембрану возможен и их взаимодействие с сульфогруппами мембраны исключает часть протонов из ионного обмена. Кроме того, согласно данным статьи [22], при относительно невысокой концентрации группы R1 часть сульфогрупп мембраны не участвует в ионном обмене из-за образования водородных связей $\left(\mathrm{NH}_{\mathrm{x}}{ }^{+} \ldots \mathrm{SO}_{3}{ }^{-}\right)$.

Для определения ионов $\mathrm{ProH}^{+}$и $\mathrm{LidH}^{+}$в комбинированном препарате была выбрана пара мембран Nafion (экструзия, in situ) +2.4 мас.\% $\mathrm{ZrO}_{2}$ и $4 \mathrm{CK}_{\text {(отливка) }}+3$ мас.\% $\mathrm{SiO}_{2}$ (10 мол.\% R1), одна из которых обеспечивает максимальную чувствительность ПД-сенсора к ионам $\mathrm{ProH}^{+}$, другая - к ионам $\mathrm{LidH}^{+}$, а корреляция между откликами ПД-сенсоров на их основе минимальна. Система градуировочных уравнений ПД-сенсоров для совместного определения ионов $\mathrm{ProH}^{+}$и $\mathrm{LidH}^{+}$в диапазоне концентраций $1.0 \cdot 10^{-4}-1.0 \cdot 10^{-2} \mathrm{M}(\mathrm{pH}$ 4.06-6.09) имеет вид:

$$
\left\{\begin{array}{l}
\Delta \varphi_{\mathrm{D}}=-68-25.8 \cdot \mathrm{pProH}+10.4 \cdot \mathrm{pLidH}-5.9 \cdot \mathrm{pH} \\
\Delta \varphi_{\mathrm{D}}=245-31.2 \cdot \mathrm{pProH}-41.9 \cdot \mathrm{pLidH}-27.0 \cdot \mathrm{pH} .
\end{array}\right.
$$

Рассчитанные пределы обнаружения ионов $\mathrm{ProH}^{+}$и $\mathrm{LidH}^{+}$в присутствии ионов $\mathrm{H}_{3} \mathrm{O}^{+}$с помощью выбранной пары перекрестно чувствительных ПД-сенсоров, а также исходные данные для их оценки представлены в табл. 1.

Таблица 1. Пределы обнаружения ионов $\mathrm{ProH}^{+}$и $\mathrm{LidH}^{+}$в присутствии $\mathrm{H}_{3} \mathrm{O}^{+}$с помощью системы ПД-сенсоров на основе мембран Nafion+2.4 мас.\% $\mathrm{ZrO}_{2}$ и МФ-4СК+3 мас.\% $\mathrm{SiO}_{2}(10$ мол.\% R1)

\begin{tabular}{|c|c|c|c|c|}
\hline Состав мембраны & $\left(\Delta \varphi_{\mathrm{D}}\right)_{\text {фон }}, \mathrm{MB}$ & $\mathrm{s}_{\text {фон }}, \mathrm{MB}$ & $\mathrm{c}_{\min }\left(\mathrm{ProH}^{+}\right), \mathrm{M}$ & $\mathrm{c}_{\min }\left(\mathrm{LidH}^{+}\right), \mathrm{M}$ \\
\hline Nafion+2.4 мас.\% $\mathrm{ZrO}_{2}$ & 223 & 13 & \multirow{2}{*}{$1.0 \cdot 10^{-6}$} & \multirow{2}{*}{$4.5 \cdot 10^{-5}$} \\
\hline 3Ф-4CК+ & 196 & 30 & & \\
\hline
\end{tabular}

Таблица 2. Результаты определения ионов $\mathrm{ProH}^{+}$и $\mathrm{LidH}^{+}$в комбинированном препарате с помощью мультисенсорной системы на основе мембран Nafion + 2.4 мас.\% $\mathrm{ZrO}_{2}$ и МФ-4СК + 3 мас.\% $\mathrm{SiO}_{2}$ (10 мол.\% R1) (n=5, p=0.95)

\begin{tabular}{|c|c|c|c|c|c|c|c|c|c|}
\hline \multirow[b]{2}{*}{$\mathrm{pH}$} & \multirow{2}{*}{$\begin{array}{c}\mathrm{V}_{\text {«Новокаин»»}} / \\
\mathrm{V}_{\text {«Лидокаин» }} / \\
\mathrm{V}_{\text {раствора }}, \mathrm{cm}^{3}\end{array}$} & \multicolumn{2}{|c|}{$\mathrm{c}_{\text {препарат }}, \mathrm{M}$} & \multicolumn{2}{|c|}{$\overline{\mathrm{c}}_{\text {эксп }}, \mathrm{M}$} & \multicolumn{2}{|c|}{$\delta, \%$} & \multicolumn{2}{|c|}{$\mathrm{s}_{\mathrm{r}}, \%$} \\
\hline & & 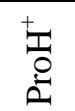 & 吾 & $\begin{array}{l}+ \\
+1 \\
O \\
0 \\
0\end{array}$ & 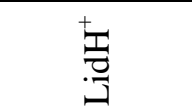 & 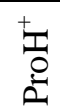 & 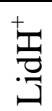 & 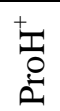 & 五 \\
\hline $4.68 \pm 0.03$ & $0.7 / 1.4 / 50$ & $\begin{array}{c}1.0 \\
.10^{-3}\end{array}$ & $\begin{array}{l}1.0 \\
.10^{-2}\end{array}$ & $\begin{array}{c}(1.01 \pm 0.08) \\
\cdot 10^{-3}\end{array}$ & $\begin{array}{c}(1.05 \pm 0.10) \\
\cdot 10^{-2}\end{array}$ & 0.7 & 5 & 8 & 10 \\
\hline $4.33 \pm 0.03$ & $7 / 0.14 / 50$ & $\begin{array}{l}1.0 \\
.10^{-2}\end{array}$ & $\begin{array}{l}1.0 \\
.10^{-3}\end{array}$ & $\begin{array}{c}(1.01 \pm 0.11) \\
\cdot 10^{-2}\end{array}$ & $\begin{array}{c}(0.94 \pm 0.12) \\
\cdot 10^{-3}\end{array}$ & 0.5 & 6 & 11 & 12 \\
\hline $6.09 \pm 0.05$ & $0.07 / 0.014 / 50$ & $\begin{array}{l}1.0 \\
.10^{-4}\end{array}$ & $\begin{array}{l}1.0 \\
.10^{-4}\end{array}$ & $\begin{array}{c}(1.11 \pm 0.04) \\
\cdot 10^{-4}\end{array}$ & $\begin{array}{c}(0.95 \pm 0.12) \\
\cdot 10^{-4}\end{array}$ & 11 & 5 & 4 & 12 \\
\hline $5.53 \pm 0.12$ & $0.7 / 0.014 / 50$ & $\begin{array}{l}1.0 \\
.10^{-3}\end{array}$ & $\begin{array}{l}1.0 \\
.10^{-4}\end{array}$ & $\begin{array}{c}(1.10 \pm 0.06) \\
\cdot 10^{-3}\end{array}$ & $\begin{array}{c}(0.94 \pm 0.11) \\
\cdot 10^{-4}\end{array}$ & 10 & 6 & 6 & 11 \\
\hline
\end{tabular}

В табл. 2 представлены величины рН, заявленные и найденные значения концентраций ионов $\mathrm{ProH}^{+}$и $\mathrm{LidH}^{+}$в растворах препарата, приготовленных из растворов для инъекций в их различном объемном соотношении с последующим разбавлением бидистиллированной водой. Относительная погрешность и относительное стандартное отклонение определения ионов $\mathrm{ProH}^{+}$составили 0.5-11 и 4-11\%, ионов $\mathrm{LidH}^{+}-5-6$ и 10-12\%, соответственно. 


\section{Заключение}

Исследовано влияния способа получения перфторированных сульфокатионообменнных мембран и гибридных материалов на их основе на характеристики ПД-сенсоров в растворах гидрохлоридов прокаина и лидокаина. Показано, что различия значений чувствительности ПД-сенсоров к катионам прокаина и лидокаина, связанные с различиями их размера и гидрофильности, могут быть использованы для их совместного определения в водных растворах. При этом модификация мембран допантами с протоноакцепторными свойствами позволяет снизить чувствительность ПДсенсоров к ионам $\mathrm{H}_{3} \mathrm{O}^{+}$, мешающим определению катионов местных анастетиков. Предложена мультисенсорная система на основе мембран МФ-4СК и Nafion, содержащих 3 мас.\% $\mathrm{SiO}_{2}$, поверхностно модифицированного 10 мол.\% 3-аминопропила, и 2.4 мас.\% $\mathrm{ZrO}_{2}$ для определения катионов прокаина и лидокаина в диапазоне концентраций от $1.0 \cdot 10^{-4}$ до $1.0 \cdot 10^{-2} \mathrm{M}$. Пределы обнаружения ионов прокаина и лидокаина с помощью предложенной мультисенсорной системы составили $1.0 \cdot 10^{-6}$ и $4.5 \cdot 10^{-5} \mathrm{M}$, соответственно. Относительная погрешность и относительное стандартное отклонение определения катионов прокаина в комбинированном фармацевтическом препарате составили 0.5-11 и 4-11 \%, катионов лидокаина - 5-6 и 10-12\%, соответственно.

Исследование выполнено при финансовой поддержке РФФИ

и Правительства Воронежской области в рамках научного проекта № 19-48-363008.

\section{Список литературы}

1. Машковский М.Д. Лекарственные средства. М. Новая волна. 2005. 1200 с.

2. Qin W.W., Jiao Z., Zhong M.K., Shi X.J. et al. // Journal of Chromatography B. 2010. Vol. 878. No 15-16. pp. 1185-1189. DOI: 10.1016/j.jchromb.2010.03.003.

3. Murtaza R., Jackman H.L., Alexander B., Lleshi-Tali A. et al. // Journal of pharmacological and toxicological methods. 2001. Vol. 46. No 3. pp. 131-136. DOI: 10.1016/S1056-8719(02)00171-5.

4. Tanaka E., Nakamura T., Inomata S., Honda K. // Journal of Chromatography B. 2006. Vol. 834. No 1-2. pp. 213-216. DOI: 10.1016/j.jchromb.2006.02.034.

5. Koehler A., Oertel R., Kirch W. // Journal of Chromatography A. 2005. Vol. 1088. No 1-2. $\quad$ pp. 126-130. DOI: 10.1016/j.chroma.2005.03.071.

6. Daryanavard S.M., Jeppsson-Dadoun A., Andersson L.I., Hashemi M. et al. // Biomedical Chromatography. 2013. Vol. 27. No 11. pp. 1481-1488. DOI: 10.1002/bmc.2946.

7. Baniceru M., Croitoru O., Popescu S.M. // Journal of pharmaceutical and biomedical analysis. 2004. Vol. 35. No 3. pp. 593-598. DOI: 10.1016/j.jpba.2004.02.012.
8. Youngvises N., Liawruangrath B., Liawruangrath S. // Journal of pharmaceutical and biomedical analysis. 2003. Vol. 31. No 4. pp. 629-638. DOI: 10.1016/S0731-7085(02)006933.

9. Ashour S., Boudakji A., Alfares B. // Res. J. of Aleppo. Univ. 2009. Vol. 64. No 5. pp. 52-63.

10. Omer L.S., Ali R.J. // International Journal of Chemistry. 2017. Vol. 9. No 4. pp. 49-61. DOI: 10.5539/ijc.v9n4p49.

11. Amin A.S., El-Didamony A.M. // Analytical sciences. 2003. Vol. 19. No 10. pp. 1457-1459. DOI: 10.2116/analsci.19.1457.

12. Michalska K., Pajchel G., Tyski S. // Journal of Chromatography B. 2004. Vol. 800. No 1-2. pp. 203-209. DOI: 10.1016/j.jchromb.2003.10.017.

13. Liu A.L., Wang J.D., Chen W., Xia X.H. et al. // Journal of Solid State Electrochemistry. 2012. Vol. 16. No 4. pp. 1343-1351. DOI: 10.1007/s10008-011-1517-2.

14. Bergamini M.F., Santos A.L., Stradiotto N.R., Zanoni M.V.B. // Journal of pharmaceutical and biomedical analysis. 2007. Vol. 43. No 1. pp. 315-319. DOI: 10.1016/j.jpba.2006.06.001. 
15. Кулапина Е.Г., Баринова О.В. // Журнал аналитической химии. 2001. Т. 56. №. 5. C. 518-522.

16. Yang C., Denno M. E., Pyakurel P., Venton B.J. // Analytica chimica acta. 2015. Vol. 887. pp. 17-37. DOI: 10.1016/j.aca.2015.05.049.

17. Abo-Hamad A., AlSaadi M.A., Hayyan M., Juneidi I. et al // Electrochimica Acta. 2016. Vol. 193. pp. 321-343. DOI: 10.1016/j.electacta.2016.02.044.

18. Oliveira R.T., Salazar-Banda G.R., Ferreira V.S., Oliveira S.C. et al. // Electroanalysis: An International Journal Devoted to Fundamental and Practical Aspects of Electroanalysis. 2007. Vol. 19. No 11. pp. 1189-1194. DOI: 10.1002/elan.200603840.

19. Komorsky-Lovrić Š., Vukašinović N., Penovski R. // Electroanalysis: An Internation-

\section{References}

1. Mashkovskiy M.D. Lekarstvennyye sredstva, M., Novaya volna, 2005, 1200 p.

2. Qin W.W., Jiao Z., Zhong M.K., Shi X. J., Zhang J. et al., Journal of Chromatography B, 2010, Vol. 878, No 15-16, pp. 1185-1189. DOI: 10.1016/j.jchromb.2010.03.003.

3. Murtaza R., Jackman H.L., Alexander B., Lleshi-Tali A. et al., Journal of pharmacological and toxicological methods, 2001, Vol. 46, No 3, pp. 131-136. DOI: 10.1016/S10568719(02)00171-5.

4. Tanaka E., Nakamura T., Inomata S., Honda K., Journal of Chromatography B, 2006, Vol. 834, No 1-2, pp. 213-216. DOI: 10.1016/j.jchromb.2006.02.034.

5. Koehler A., Oertel R., Kirch W., Journal of Chromatography A, 2005, Vol. 1088, No 12 , pp. 126-130. DOI: 10.1016/j.chroma. 2005.03.071.

6. Daryanavard S.M., Jeppsson-Dadoun A., Andersson L.I., Hashemi M. et al., Biomedical Chromatography, 2013, Vol. 27, No 11, pp. 1481-1488. DOI: 10.1002/bmc.2946.

7. Baniceru M., Croitoru O., Popescu S.M., Journal of pharmaceutical and biomedical analysis, 2004, Vol. 35, No 3, pp. 593-598. DOI: 10.1016/j.jpba.2004.02.012.

8. Youngvises N., Liawruangrath B., Liawruangrath S., Journal of pharmaceutical and biomedical analysis, 2003, Vol. 31, No 4, pp. 629-638. DOI: 10.1016/S0731-7085(02)006933. al Journal Devoted to Fundamental and Practical Aspects of Electroanalysis. 2003. Vol. 15. No 5-6. pp. 544-547. DOI: 10.1002/elan.200390067.

20. Бобрешова О.В., Паршина А.В., Сафронова Е.Ю., Янкина К.Ю. и др. // Журнал аналитической химии. 2015. Т. 70. № 5. С. 543-549.

21. Ярославцев А.Б. // Высокомолекулярные соединения. Сер. А. 2013. Т. 55. № 11. C. 1367.

22. Михеев А.Г., Сафронова Е.Ю., Ярославцев А.Б. // Мембраны и мембранные технологии. 2013. Т. 3. № 2. С. 93-99.

23. Паршина А.В., Бобрешова О.В., Денисова Т.С. // Сорбиионные и хроматографические процессы. 2015. Т.15. № 6. С. 794801. DOI: 10.17308/sorpchrom.2015.15/333.

9. Ashour S., Boudakji A., Alfares B., Res. J. of Aleppo. Univ, 2009, Vol. 64, No 5, pp. 5263.

10. Omer L.S., Ali R.J., International Journal of Chemistry, 2017, Vol. 9, No 4, pp. 49-61. DOI: 10.5539/ijc.v9n4p49.

11. Amin A.S., El-Didamony A.M., Analytical sciences, 2003, Vol. 19, No 10, pp. 1457-1459. DOI: 10.2116/analsci.19.1457.

12. Michalska K., Pajchel G., Tyski S., Journal of Chromatography B, 2004, Vol. 800, No 1-2, pp. 203-209. DOI: 10.1016/j.jchromb.2003.10.017.

13. Liu A.L., Wang J.D., Chen W., Xia X.H. et al., Journal of Solid State Electrochemistry, 2012, Vol. 16, No 4, pp. 1343-1351. DOI: $10.1007 / \mathrm{s} 10008-011-1517-2$.

14. Bergamini M.F., Santos A.L., Stradiotto N.R., Zanoni M.V.B., Journal of pharmaceutical and biomedical analysis, 2007, Vol. 43, No 1, pp. 315-319. DOI: 10.1016/j.jpba.2006.06.001.

15. Kulapina E.G., Barinova O.V., Journal of Analytical Chemistry, 2001, Vol. 56, No 5, pp. 457-460. DOI: 10.1023/A:1016683121191

16. Yang C., Denno M. E., Pyakurel P., Venton B.J., Analytica chimica acta, 2015, Vol. 887, pp. 17-37. DOI: 10.1016/j.aca.2015.05.049.

17. Abo-Hamad A., AlSaadi M.A., Hayyan M., Juneidi I. et al., Electrochimica 
Acta, 2016, Vol. 193, pp. 321-343. DOI: 10.1016/j.electacta.2016.02.044.

18. Oliveira R.T., Salazar-Banda G.R., Ferreira V.S., Oliveira S.C. et al., Electroanalysis: An International Journal Devoted to Fundamental and Practical Aspects of Electroanalysis, 2007, Vol. 19, No 11, pp. 11891194. DOI: 10.1002/elan.200603840.

19. Komorsky-Lovrić Š., Vukašinović N., Penovski R., Electroanalysis: An International Journal Devoted to Fundamental and Practical Aspects of Electroanalysis, 2003, Vol. 15, No 5-6, $\mathrm{pp}$. 544-547. 10.1002/elan.200390067.

Паршина Анна Валерьевна - д.Х.н., доцент, кафедра аналитической химии, Воронежский государственный университет, Воронеж

Колганова Татьяна Сергеевна - преподаватель, кафедра аналитической химии, Воронежский государственный университет, Воронеж

Бобрешова Ольга Владимировна - д.х.н., профессор, главный научный сотрудник, кафедра аналитической химии, Воронежский государственный университет, Воронеж
20. Bobreshova O.V., Parshina A.V., Safronova E.Yu., Yankina K.Yu. et al., Journal of Analytical Chemistry, 2015, Vol. 70, No 5, pp. 627-632. DOI: 10.1134/S1995078013060049.

21. Yaroslavtsev A.B., Polymer Science Series A, 2013, Vol. 55, No 11, pp. 674-698. DOI: 10.1134/S0965545X13110060.

22. Mikheev A.G., Safronova E.Y., Yaroslavtsev A.B., Petroleum Chemistry, 2013, Vol. 53, No 7, pp. 504-510. DOI: 10.1134/S0965544113070104.

23. Parshina A.V., Bobreshova O.V., Denisova T.S., Sorbtsionnyye i khromatograficheskiye protsessy, 2015, Vol. 15, No 6, pp. 794-801.

Parshina Anna V. - Dr.Sci. (Chemistry), docent, Department of Analytical Chemistry, Voronezh State University, Voronezh parshina_ann@mail.ru

Kolganova Tatyana S. - teacher, Department of Analytical Chemistry, Voronezh State University, Voronezh

Bobreshova Olga V. - Dr.Sci. (Chemistry), professor, chief researcher, Department of Analytical Chemistry, Voronezh State University, Voronezh 